\title{
Predictores individuales, contextuales y organizacionales de engagement en el trabajo y job crafting
}

\author{
Individual, contextual and organizational predictors of work engagement and job \\ crafting
}

\section{Preditores individuais, contextuais e organizacionais de engajamento no trabalho e job crafting}

\author{
Jhoanna Altamirano ${ }^{1}$, ORCID 0000-0002-5136-9700 \\ Paula Oviedo $^{2}$, ORCID 0000-0003-1771-0247 \\ Bruna Simões Tocchetto ${ }^{3}$, ORCID 0000-0001-9141-8370 \\ Manoela Ziebell de Oliveira ${ }^{4}$, ORCID 0000-0003-0243-5115 \\ ${ }^{124}$ Pontificia Universidad Católica de Río Grande del Sur (PUCRS). Brasil \\ ${ }^{3}$ Fundación Universidad Federal de Ciencias de la Salud de Porto Alegre (UFCSPA). Brasil
}

\begin{abstract}
Resumen: El propósito de esta investigación fue identificar, en un grupo de motivadores, los predictores de engagement en el trabajo y comportamientos de job crafting con base en la teoría de demandas y recursos laborales junto con el modelo de las ocho fuerzas motivacionales de permanencia y turnover. Integraron la muestra 215 trabajadores brasileños del área de comunicación y marketing. Los resultados mostraron que: a) los aspectos individuales no predicen engagement ni job crafting, b) los aspectos contextuales (expectativas de amigos y empleabilidad) predicen de forma diferenciada ambas variables; y finalmente, c) los aspectos organizacionales (identificación afectiva, posibilidades de crecimiento, relaciones interpersonales y costos asociados a dejar la organización) predicen positivamente tanto engagement como job crafting. Considerando los beneficios de ambas variables a nivel individual y organizacional, el estudio contribuye a la literatura del área al destacar elementos que las promueven.
\end{abstract}

Palabras clave: engagement laboral, job crafting, comportamiento organizacional, motivación

Abstract: The purpose of this research was to identify, in a group of motivators, the predictors of engagement at work and job crafting based on the job demand-resources theory with the eight motivational forces model of permanence and turnover. The sample comprised 215 Brazilian workers of the communication and marketing area. The results showed that: a) the individual aspects do not predict engagement at work nor job crafting, b) the contextual aspects (friends expectations and employability) predict in a different way both variables; and finally, c) the organizational aspects (affective identification, growth possibilities, interpersonal relationships and costs associated with leaving the organization) positively predict both engagement at work and job crafting. Considering the benefits of both variables at the individual and organizational level, the study contributes to the literature of the area by highlighting elements that promote them.

Keywords: work engagement, job crafting, organizational behavior, motivation 
Resumo: O objetivo deste estudo foi identificar, a partir de um grupo de motivadores, os preditores de engajamento no trabalho e comportamentos de job crafting com base na teoria de demandas e recursos de trabalho e no modelo das oito forças motivacionais de permanência e turnover. A amostra foi composta por 215 trabalhadores brasileiros da área de comunicação e marketing. Os resultados mostraram que: a) os aspectos individuais não predizem engajamento e job crafting; b) os aspectos contextuais (expectativas de amigos e empregabilidade) predizem de forma diferenciada ambas as variáveis; e por fim, c) os aspectos organizacionais (identificação afetiva, possibilidades de crescimento, relações interpessoais e custos associados a deixar a organização) predizem positivamente tanto engajamento como job crafting. Considerando os benefícios de ambas variáveis em relação aos níveis individual e organizacional, o estudo contribui para a literatura da área ao destacar elementos que as promovem.

Palavras-chave: engajamento no trabalho, job crafting, comportamento organizacional, motivação

Aceptado: 21/05/2020

Cómo citar:

Altamirano, J., Oviedo, P., Tocchetto, B.S., \& Oliveira, M.Z. (2020). Predictores individuales, contextuales y organizacionales de engagement en el trabajo y job crafting. Ciencias Psicológicas, 14(2), e-2202. doi: https://doi.org/10.22235/cp.v14i2.2202

Correspondencia: Jhoanna Altamirano; PUCRS. Av. Ipiranga, 6681, Prédio 11, Sala 938. CEP 90619-900. Porto Alegre - RS, Brasil. E-mail: jhoanna.altamirano.b@gmail.com. Paula Oviedo, PUCRS. E-mail: paula.ferreira.rp@gmail.com. Bruna Simões Tocchetto, UFCSPA. Calle Sarmento Leite, 245, anexo II, sala 117. CEP 90050-170. Porto Alegre - Rs, Brasil. E-mail: br.tocchetto@gmail.com. Dra. Manoela Ziebell de Oliveira, PUCRS. E-mail: manoela.ziebell@gmail.com

El mundo laboral se encuentra en constante cambio. Esto se refleja en las dinámicas internas de trabajo y también en la forma en cómo las personas comprenden y dirigen sus propias carreras, dando cada vez más valor a las experiencias positivas, priorizando los objetivos individuales y buscando una vida laboral saludable y satisfactoria. Es así que las carreras ya no son determinadas únicamente por demandas organizacionales, sino también por los objetivos de las personas (Demerouti, 2014). Si antes el foco de gestión era la productividad, desde algunos años cada vez más empresas invierten en acciones de gestión de recursos humanos, como ejercicios de retroalimentación, promoción para participación en la toma de decisiones, soporte por parte de los líderes y facilidad para la variedad de tareas (Bakker \& Schaufeli, 2008).

Ante estas cuestiones, las organizaciones enfrentan dificultades tanto para captar como para retener colaboradores (Oliveira, Beria, \& Gomes, 2016), evidenciando cierta incapacidad para administrar el factor humano, y sus exigencias individuales, tiempo que se atienden los objetivos del negocio. En contraparte, los trabajadores se perciben cada vez más desafiados por las demandas de sus corporaciones (Bakker \& Demerouti, 2016), las cuales esperan altos niveles de productividad y valorizan otras capacidades como flexibilidad, trabajo en equipo, compromiso, proactividad y aprendizaje constante (Bakker \& Schaufeli, 2008; Hakanen, Peeters, \& Schaufeli, 2017).

El área de comunicación es un claro ejemplo de esta problemática. Las grandes empresas de marketing y propaganda vienen enfrentando un movimiento de migración de sus trabajadores, principalmente de los más jóvenes, para otros modelos de negocios relacionados al emprendimiento (Carvalho, Alves \& Machado, 2016). Incentivados por los constantes cambios 
en el mercado, los trabajadores de comunicación buscan más que un empleo, un espacio que les permita satisfacer sus necesidades como profesionales e individuos (Carvalho et al., 2016). Por esto, la comunicación deja de ser un área que posee un bajo histórico de atención al capital humano y comienza a mirar a las nuevas demandas de sus profesionales (Carvalho \& Christofoli, 2015).

La complejidad de estos fenómenos requiere un análisis con foco en las experiencias, características individuales y aspectos positivos, diferenciándose del paradigma psicológico tradicional y explorando las cualidades relacionadas al ser humano en el ambiente organizacional, por lo que nos adentramos en esta dinámica laboral desde la perspectiva de la Teoría de Demandas y Recursos Laborales (DRL; Demerouti, Bakker, Nachreiner, \& Schaufeli, 2001). Este modelo teórico sugiere que las características de todo trabajo pueden agruparse en dos categorías: demandas laborales y recursos laborales. A través de la interacción de estos, junto con los recursos personales, se dan los procesos de motivación y de deterioro laboral (Bakker \& Demerouti, 2014). Adicionalmente, la teoría propone que la relación entre las características laborales y la salud y bienestar del trabajador se influencian mutuamente a través del tiempo (Bakker \& Demerouti, 2014).

Uno de los indicadores de bienestar individual más estudiados por esta teoría es engagement (Bakker, Demerouti \& Sanz-Vergel, 2014), por su impacto positivo tanto para las organizaciones como para los propios trabajadores (Bakker, Schaufeli, Leiter \& Taris, 2008). Engagement es definido como el estado mental positivo de satisfacción relacionado al trabajo caracterizado por vigor, dedicación y absorción (Schaufeli, Bakker, \& Salanova, 2006). Concretamente es cuando un individuo tiene altos niveles de energía y resiliencia mental, demostrando disposición a invertir esfuerzos en el propio trabajo y persiste frente a dificultades; es involucrarse fuertemente con sus actividades y encontrar estas significativas, sentirse entusiasmado e inspirado en el trabajo llegando inclusive a alcanzar estados de completa concentración y absorción (Wingerden, Bakker, \& Derks, 2016). Este estado se relaciona con diversos resultados organizacionales positivos. Trabajadores con más engagement son más innovadores (Hakanen, Perhoniemi, \& Toppinen-Tammer, 2008), experimentan un mejor ajuste con relación a sus trabajos (Lascbinger, Wong, \& Greco, 2006), tienen altos niveles de desempeño (Bakker \& Bal, 2010), son más creativos (Bakker \& Xanthopoulou, 2013) y muestran comportamientos de ciudadanía organizacional (Rich, Lepine, \& Crawford, 2010). Engagement es importante también ya que puede promover el bienestar y la salud (Airila et al., 2014; Hakanen \& Schaufeli, 2012). Estudios demuestran además que engagement está asociado a comportamientos proactivos (Macey \& Schneider, 2008), ya que siendo un estado de bienestar motivacional con altos niveles de energía (Salanova, Schaufeli, Xanthopoulou \& Bakker, 2010), se relaciona intrínsecamente con la adopción de comportamientos autodireccionados. Es así que diversos estudios ya han centrado su foco en la relación de esta variable con job crafting (Bakker, Rodríguez-Muñoz, \& Sanz-Vergel, 2016; Demerouti, 2014).

Job crafting es un término acuñado por Wrzesniewski y Dutton (2001) para definir los cambios físicos y cognitivos de iniciativa propia donde los individuos alteran sus trabajos con la finalidad de alinear este con las habilidades, necesidades y preferencias propias. De acuerdo a la teoría de demandas y recursos laborales, se distinguen cuatro tipos de job crafting: incremento de recursos estructurales (i.e., buscar formas de desarrollarse uno mismo como profesional o tratar de aprender cosas nuevas), incremento de recursos sociales (i.e., solicitar retroalimentación sobre el desempeño propio tanto a colegas como a supervisores), incremento de demandas desafiantes (i.e., involucrarse de forma voluntaria en proyectos o tareas nuevas) y reducción de demandas obstaculizantes (i.e., evitar o eliminar contacto con situaciones mental o emocionalmente desgastantes). Los tres primeros pueden ser clasificados como job crafting de tipo expansivo (Wrzesniewski \& Dutton, 2001), mientras que el ultimo debe ser considerado más próximo a una estrategia de afrontamiento (Hakanen, Peeters \& Schaufeli, 2017). Esto se debe a que algunas 
demandas laborales tienen únicamente impactos negativos en los trabajadores, por lo que la disminución de estas demandas obstaculizantes es un mecanismo de protección de la salud del propio trabajador (Demerouti, 2014). Es así que job crafting es un tipo de rediseño de trabajo de abajo hacia arriba, que debe considerarse una alternativa complementaria a los enfoques de arriba hacia abajo (Demerouti, 2014) ya que aumenta el número de recursos laborales e impacta en el bienestar general de los trabajadores (Tims, Bakker \& Derks, 2013). Este comportamiento organizacional está relacionado también con el incremento del desempeño (Demerouti, Bakker, \& Gevers, 2015), permite que los individuos resignifiquen sus trabajos y aumenten las probabilidades de establecer mejores relaciones, al mismo tiempo que alcanzan sus objetivos individuales (Slemp \& Vella-Brodrik, 2014). El presente estudio no ahondará más en la ya bien establecida relación entre engagement y job crafting (Demerouti, 2014), sino que nos enfocaremos en identificar qué factores las predicen de forma independiente.

Adicionalmente a los enfoques que estudian las cualidades individuales y aspectos positivos dentro del plano laboral, los fenómenos de rotación, vienen impulsando a gestores e investigadores a preguntarse exactamente por qué motivos los trabajadores permanecen o dejan sus empleos (Holtom, Mitchell, Lee \& Eberly, 2008). Uno de los estudios que representa estos esfuerzos teóricos resultó en el modelo de las ocho fuerzas motivacionales para permanencia y turnover (rotación) de Maertz y Griffeth (2004). Este modelo integral considera aspectos de tres niveles: individuales, contextuales y organizacionales, traducidos en fuerzas motivacionales (moral, normativa, alternativa, afectiva, calculativa, contractual, constituyente y comportamental).

Uno de los aspectos individuales contemplados en el modelo es la fuerza moral, que son los valores y creencias favorables o desfavorables sobre salir y/o permanecer en la organización (Maertz \& Griffeth, 2004). Si bien no se encontró literatura que relacione directamente estas creencias con engagement o job crafting, podemos suponer que ambos (estado y comportamiento) pueden verse influenciados por los valores individuales, que juegan un papel fundamental moldeando objetivos y comportamientos (Shin \& Zhou, 2003). Así, las hipótesis 1 y 2 son:

Hipótesis 1: Las fuerzas morales, a) pro-vínculo y b) pro-rotación, predicen positiva y negativamente Engagement.

Hipótesis 2: Las fuerzas morales, a) pro-vínculo y b) pro-rotación, predicen positiva y negativamente Job crafting.

Los aspectos contextuales están determinados por las fuerzas normativas y alternativas. La fuerza normativa muestra cómo el propio individuo percibe las expectativas de personas fuera de su trabajo (familia y amigos) sobre su continuidad en la organización actual (Maertz \& Griffeth, 2004). A pesar de las diversas investigaciones sobre los efectos positivos de engagement en el trabajo, su relación con aspectos familiares del individuo aún es poco abordados (Culbertson, Mills \& Fullagar, 2012) así como su relación con otros círculos cercanos. La fuerza alternativa por su parte es un reflejo de la interacción entre la propia capacidad percibida para encontrar un nuevo trabajo y la percepción que se tiene sobre el mercado actual (Maertz \& Griffeth, 2004; Oliveira et al., 2016). Con respecto a la empleabilidad percibida, la literatura muestra que sentirse capaz de adquirir nuevas alternativas laborales aumenta el sentido de bienestar y la percepción de éxito objetivo y subjetivo (Guest \& Rodrigues, 2015). Así, se establecen las siguientes hipótesis:

Hipótesis 3: La fuerza a) normativa predice negativamente Engagement y la fuerza b) alternativa la predice de forma positiva.

Hipótesis 4: La fuerza a) normativa predice negativamente Job crafting y la fuerza b) alternativa la predice de forma positiva.

Por último, el modelo de motivos para permanencia y turnover contempla también los aspectos organizacionales. Maertz y Griffeth (2004) categorizan estos en cinco fuerzas: a) 
afectiva, es una tendencia motivacional que implica emociones positivas generadas por la organización y por ser parte de esta, teniendo en consideración que lo que piense el trabajador sobre la organización va a generar una respuesta emocional; b) calculativa, involucra la evaluación racional sobre las posibilidades de desarrollo en la organización; c) contractual, comprende percepciones sobre qué es lo que la organización le debe al trabajador, y de forma inversa lo que este le debe, donde el sentido de obligación a la permanencia es una respuesta a cuestiones relacionadas al contrato psicológico estipulado al inicio del vínculo laboral; d) constituyente, trata sobre la calidad de las relaciones con los líderes y compañeros de trabajo; e) comportamental, expresa los costos asociados percibidos sobre dejar la organización actual.

Estudios previos muestran que diversos aspectos relacionados con estas fuerzas, como el soporte social, el control sobre el propio trabajo, la oportunidad de aprendizaje y la retroalimentación sobre el desempeño tienen efectos positivos sobre engagement en el trabajo (Bakker, Schaufeli, Leiter, \& Taris, 2008). Del mismo modo, las expectativas generadas por las condiciones implícitas y explicitas de los contratos laborales pueden generar sentimientos de violación ya que el individuo va esperar trato justo y mutualidad (Rios, Lula, Amaral, \& Bastos, 2014). A su vez, algunos estudios identifican al clima organizacional (Scott \& Bruce, 1994), las relaciones interpersonales (Janssen, Van de Vliert \& West, 2004) y a las características de trabajo (Oldhman \& Cummings, 1996) como antecedentes importantes de comportamientos innovadores. Si bien job crafting es un constructo diferente, recientes estudios sugieren que los trabajadores modifican sus trabajos de acuerdo a las percepciones que estos tienen sobre el mismos (Hakanen et al., 2017). Es así que se establecen las siguientes hipótesis:

Hipótesis 5: Las fuerzas a) afectiva, b) calculativa, c) constituyente y d) comportamental y el e) componente cumplimento de la fuerza contractual predicen positivamente engagement, mientras que el f) componente descumplimiento de la fuerza contractual lo predice de forma negativa.

Hipótesis 6: Las fuerzas a) afectiva, b) calculativa, c) constituyente y d) comportamental y el e) componente cumplimento de la fuerza contractual predicen positivamente Job crafting, mientras que el f) componente descumplimiento de la fuerza contractual lo predice de forma negativa.

En este contexto de constantes cambios, donde la retención del factor humano y la promoción de aspectos positivos se convierten en el principal foco de interés tanto para profesionales como para académicos, se ve necesario entender qué situaciones específicas tienen potencial de estimular o promover el engagement en el trabajo y los comportamientos de job crafting. El presente estudio, por tanto, objetiva explorar qué aspectos individuales, contextuales y organizacionales influencian engagement y job crafting.

\section{Método}

\section{Participantes y procedimientos}

La muestra final resultó en 215 trabajadores brasileños del sector de comunicación y marketing de la ciudad de Porto Alegre. Con una edad media de 32.1 años $(D E=8.71)$ y $61.4 \%$ eran mujeres. Respecto al tiempo de permanencia en la organización, 56.7\% tenía menos de dos años, $26.5 \%$ entre dos y cinco años once meses, y $16.7 \%$ más de seis años. El $55.3 \%$ contaban con estudios universitarios, $26 \%$ con educación básica regular y un $18.6 \%$ con especialización o posgrado.

Los investigadores contactaron nueve agencias de comunicación y marketing a través del sindicato de la región. En reuniones con las áreas de gestión humana de cada agencia se explicaron los objetivos y se solicitó permiso para la colecta de información, seis de estas instituciones aceptaron participar del estudio. Luego, se realizaron reuniones con los 
colaboradores para presentar la investigación, resolver dudas y explicar el proceso de colecta. El cuestionario on-line fue divulgado vía correo electrónico, el cual contenía el término de consentimiento libre y esclarecido. Este término incluyó informaciones sobre el carácter confidencial y voluntario del estudio, así como una breve explicación de sus objetivos, procedimientos y criterios de inclusión (laborar como dependiente en el momento de la colecta, pertenecer al sector de comunicación y/o publicidad y tener como mínimo 18 años de edad). Para ser parte del estudio los participantes debían aceptar el término, indicando su conformidad marcando la opción "acepto". Se envió, entre junio y octubre de 2017, invitaciones a 385 trabajadores, y fueron respondidas satisfactoriamente 215 , alcanzando un ratio de respuesta de $55.8 \%$.

\section{Instrumentos}

Engagement fue evaluado con la escala Utrecht de Engajamento no Trabalho, versión adaptada al portugués por Vazquez, Magnan, Pacico, Hutz e Schaufeli (2015), que evalúa el estado mental general de satisfacción y disposición positiva caracterizada por vigor, dedicación y concentración (i.e. En mi trabajo me siento lleno de energía). Escala tipo Likert de siete puntos que van desde cero (nunca) a seis (siempre) con 17 frases afirmativas donde los mayores puntajes indican niveles más altos de engagement. En el estudio de adaptación la muestra brasileña tuvo una consistencia interna de $\alpha=.95 \mathrm{y}$ en el presente estudio la consistencia interna fue de $\alpha=.94$.

Job crafting se evaluó con la escala de Comportamentos de Redesenho do Trabalho, versión adaptada al portugués por Chinelato, Ferreira \& Valentini (2015). Evalúa comportamientos autoiniciados para modificar el trabajo en la forma de incremento de recursos estructurales (i.e. Intento aprender cosas nuevas), sociales (i.e. Pido a los otros feedback sobre mi desempeño) y demandas desafiantes (i.e. Cuando un proyecto interesante aparece, me ofrezco como participante de este). Escala tipo Likert de cinco puntos desde uno (nunca) a cinco (siempre) con 14 frases afirmativas, donde los mayores puntajes indican más comportamientos de job crafting. El estudio de adaptación tuvo resultados de consistencia interna de $\alpha=.71$ para incremento de recursos estructurales, $\alpha=.78$ para incremento de recursos sociales y $\alpha=.77$ para incremento de demandas desafiantes, en la presente muestra las consistencias internas fueron de $\alpha=.86, \alpha=.79$ y $\alpha=.77$ respectivamente.

Los aspectos individuales, organizacionales y contextuales fueron evaluados con el Inventário de Motivações para Turnover e Permanência, adaptado al portugués por Oliveira et al. (2016). Inventario tipo Likert que va de uno (totalmente en desacuerdo) a cinco (totalmente de acuerdo), de 88 ítems que reflejan ocho fuerzas motivacionales para permanecer o salir de la organización actual. Estas ocho fuerzas son operacionalizadas a través de 14 dimensiones, como se detalla en la Tabla 1.

Debido a que investigaciones previas sugieren que la edad, el nivel educativo, la jerarquía y el tiempo de permanencia en la organización se relacionan con nuestras variables de estudio (Berdicchia, Nicolli \& Masino, 2016; Petrou, Demerouti \& Schaufeli, 2015; Vasquez et al., 2015; Yim, Choi \& Park, 2015) fueron incorporadas preguntas concernientes a éstas, en un cuestionario sociodemográfico previo a la presentación de las escalas. 
Tabla 1.

Ocho fuerzas motivacionales para turnover y permanencia, dimensiones y valores de confiabilidad

\begin{tabular}{|c|c|c|c|}
\hline Aspectos & Fuerza / Definición & Dimensiones & $\begin{array}{l}\text { Confiabilidad estudio de adaptación / } \\
\text { muestra actual }\end{array}$ \\
\hline \multirow[t]{2}{*}{ A. individuales } & \multirow{2}{*}{$\begin{array}{l}\text { F. Moral / evalúa las actitudes hacia la permanencia en una } \\
\text { organización y hacia el cambio permanente de empleadores. }\end{array}$} & Pro vínculo & $\alpha=.63 / \alpha=.76$ \\
\hline & & Pro rotación & $\alpha=.72 / \alpha=.79$ \\
\hline \multirow[t]{9}{*}{ A. organizacionales } & $\begin{array}{l}\text { F. Afectiva / evalúa cuán positivo es el sentimiento general sobre la } \\
\text { organización y sobre ser miembro de ella. }\end{array}$ & Unidimensional & $\alpha=.89 / \alpha=.92$ \\
\hline & $\begin{array}{l}\text { F. Calculativa / evalúa la percepción sobre las posibilidades de } \\
\text { alcanzar los objetivos de carrera, de vida, y los valores personales a } \\
\text { través de la organización. }\end{array}$ & Unidimensional & $\alpha=.90 / \alpha=.86$ \\
\hline & \multirow{2}{*}{$\begin{array}{l}\text { F. Contractual / evalúa el sentido de obligación resultante del } \\
\text { cumplimiento del contrato psicológico por parte de la } \\
\text { organización. }\end{array}$} & Cumplimiento & $\alpha=.79 / \alpha=.86$ \\
\hline & & Descumplimiento & $\alpha=.89 / \alpha=.87$ \\
\hline & \multirow{5}{*}{$\begin{array}{l}\text { F. Constituyente / evalúa el vínculo y compromiso hacia super } \\
\text { y compañeros. } \\
\text { F. Comportamental / evalúa la percepción sobre los costos } \\
\text { relacionados a dejar una organización. }\end{array}$} & Compañeros & $\alpha=.71$ y .74 / $\alpha=.75$ \\
\hline & & Supervisores & $\alpha=.66$ y $.91 / \alpha=.84$ \\
\hline & & Costo psicológico & $\alpha=.64 / \alpha=.64$ \\
\hline & & Costo tangible & $\alpha=.66 / \alpha=.68$ \\
\hline & & Inercia & $\alpha=.76 / \alpha=.70$ \\
\hline \multirow[t]{3}{*}{ A. contextuales } & \multirow{3}{*}{$\begin{array}{l}\text { F. Normativa / evalúa las expectativas de familiares y amigos } \\
\text { respecto a dejar el empleo. } \\
\text { F. Alternativa / evalúa las creencias individuales sobre la propia } \\
\text { capacidad de encontrar otro empleo. }\end{array}$} & Familia & $\alpha=.81 / \alpha=.75$ \\
\hline & & Amigos & $\alpha=.80 / \alpha=.82$ \\
\hline & & Unidimensional & $\alpha=.86 / \alpha=.75$ \\
\hline
\end{tabular}

Nota. A: Aspectos, F: Fuerza. 


\section{Análisis}

Los datos fueron analizados en dos etapas. Primero se realizaron análisis de correlación de Pearson entre engagement, job crafting (incremento de recursos estructurales, incremento de recursos sociales e incremento de demandas desafiantes), los motivos para permanencia y turnover (fuerzas motivacionales categorizados en aspectos individuales, organizacionales y contextuales) y las variables sociodemográficas (edad, nivel educativo, jerarquía y tiempo de permanencia). Luego se realizaron regresiones jerárquicas controlando el efecto de las variables sociodemográficas, así mismo la inclusión de predictores se realizó de forma independiente para engagement y job crafting a través del método paso a paso.

\section{Consideraciones éticas}

Los procedimientos detallados fueron contemplados dentro los estándares éticos en investigación con seres humanos dispuesto por la Resolución del Consejo Nacional de Salud $\mathrm{N}^{\circ}$ 510/2016 de Brasil. La investigación fue aprobada por el Comité de Ética en Investigación de la Pontificia Universidad Católica de Rio Grande del Sur (Brasil) con CAAE: 66476517.7.0000.5336.

\section{Resultados}

\section{Resultados preliminares}

La Tabla 2 muestra las medias, desviaciones estándar y las correlaciones entre las variables. Estos resultados confirman la relación positiva entre engagement y todas las dimensiones de job crafting (incremento de recursos estructurales: $r=.47, p<.01$, incremento de recursos sociales: $r=.24, p<.01$ e incremento de demandas desafiantes: $r=.53, p<.01)$. Así mismo, las correlaciones de la Tabla 2 proveen soporte preliminar para las hipótesis planteadas. 
Predictores de engagement en el trabajo y job crafting

Tabla 2.

Medias, desviaciones estándar y correlaciones entre variables

\begin{tabular}{|c|c|c|c|c|c|c|c|c|c|c|c|c|c|c|c|c|c|c|c|c|c|c|c|c|}
\hline & & $M$ & $D S$ & 1 & 2 & 3 & 4 & 5 & 6 & 7 & 8 & 9 & 10 & 11 & 12 & 13 & 14 & 15 & 16 & 17 & 18 & 19 & 20 & 21 \\
\hline 1 & Edad & 32.09 & 8.71 & -- & & & & & & & & & & & & & & & & & & & & \\
\hline 2 & Nivel educativo & 1.92 & .66 & $.29 * *$ & -- & & & & & & & & & & & & & & & & & & & \\
\hline 3 & Jerarquía & 2.05 & .65 & $.39^{* *}$ & $.33^{* *}$ & -- & & & & & & & & & & & & & & & & & & \\
\hline 4 & Tiempo de permanencia & 1.60 & .76 & $.45^{* *}$ & $.17^{*}$ & $.36^{* *}$ & -- & & & & & & & & & & & & & & & & & \\
\hline 5 & Engagement & 5.14 & 1.02 & $.22 * *$ & -.02 & $.15^{*}$ & .03 & -- & & & & & & & & & & & & & & & & \\
\hline 6 & I recursos estructurales & 4.44 & .57 & -.08 & -.11 & -.03 & -.12 & $.47 * *$ & -- & & & & & & & & & & & & & & & \\
\hline 7 & I recursos sociales & 3.23 & .86 & $-.26^{* *}$ & -.08 & -.03 & $-.16^{*}$ & $.24 * *$ & $.35 * *$ & -- & & & & & & & & & & & & & & \\
\hline 8 & I demandas desafiantes & 3.64 & .74 & .10 & .01 & $.24 * *$ & .05 & $.53 * *$ & $.54 * *$ & $.39 * *$ & -- & & & & & & & & & & & & & \\
\hline 9 & F moral pro vínculo & 3.11 & .80 & $.22^{* *}$ & .08 & .07 & $.18 * *$ & $.37 * *$ & .09 & -.03 & $.16^{*}$ & -- & & & & & & & & & & & & \\
\hline 10 & F moral pro rotación & 2.75 & .73 & $-.19 * *$ & -.08 & -0.9 & -.11 & $-.33 * *$ & $-.17 *$ & -.04 & -.05 & $-.49 * *$ & -- & & & & & & & & & & & \\
\hline 11 & F normativa familia & 2.42 & .86 & -.09 & -.07 & -.00 & .00 & $-.17 *$ & $-.16^{*}$ & .03 & -.02 & -.04 & $.24 * *$ & -- & & & & & & & & & & \\
\hline 12 & F normativa amigos & 2.46 & .97 & $-.16^{*}$ & .04 & -.06 & -.01 & $-.44 * *$ & $-.22 * *$ & -.00 & -.10 & $-.27 * *$ & $.39 * *$ & $.42 * *$ & -- & & & & & & & & & \\
\hline 13 & $\mathrm{~F}$ alternativa & 4.44 & .67 & $-.21 * *$ & .08 & .09 & -.01 & .00 & $.22 * *$ & .04 & $.14 *$ & $-.18 * *$ & $.14 *$ & .04 & $.31 * *$ & -- & & & & & & & & \\
\hline 14 & $\mathrm{~F}$ afectiva & 3.86 & .89 & $.21 * *$ & -.05 & .04 & -.00 & $.72 * *$ & $.32 * *$ & $.17^{*}$ & $.28 * *$ & $.38^{* *}$ & $-.47 * *$ & $-.20 * *$ & $-.53 * *$ & -.13 & -- & & & & & & & \\
\hline 15 & F calculativa & 3.17 & .85 & .02 & -.02 & $.14 *$ & -.08 & $.62 * *$ & $.34 * *$ & $.35^{* *}$ & $.32 * *$ & $.26^{* *}$ & $-.35^{* *}$ & $-.15^{*}$ & $-.47 * *$ & $-.16^{*}$ & $.66^{* *}$ & -- & & & & & & \\
\hline 16 & F const compañeros & 3.24 & .53 & -.02 & -.09 & -.05 & -.02 & $.33 * *$ & $.18^{* * *}$ & $.24 * *$ & $.34 * *$ & $.28 * *$ & -.12 & .07 & -.10 & -.11 & $.36^{* *}$ & $.31 * *$ & -- & & & & & \\
\hline 17 & F const supervisores & 3.20 & .67 & -.06 & -.02 & -.07 & -.10 & $.38 * *$ & $.19 * *$ & $.44 * *$ & $.29 * *$ & $.22 * *$ & $-.23 * *$ & -.09 & $-.31 * *$ & $-.19 * *$ & $.50^{* *}$ & $.47 * *$ & $.47 * *$ & -- & & & & \\
\hline 18 & F comp psicológica & 3.72 & .64 & $.16^{*}$ & -.01 & .12 & .09 & $.57 * *$ & $.30 * *$ & $.21 * *$ & $.34 * *$ & $.33^{* *}$ & $-.46^{* *}$ & -.11 & $-.36^{* *}$ & -.04 & $.64^{* *}$ & $.45 * *$ & $.39 * *$ & $.43 * *$ & -- & & & \\
\hline 19 & F comp tangible & 2.35 & .69 & .10 & $-.13 *$ & .03 & .05 & $.21 * *$ & -.02 & .03 & .07 & $.33 * *$ & $-.16^{*}$ & .12 & $-.20 * *$ & $-.33 * *$ & $.18^{* *}$ & .13 & $.35^{* *}$ & $.16^{*}$ & $.25^{* *}$ & -- & & \\
\hline 20 & F comp inercia & 3.16 & .75 & .11 & -.07 & .09 & .11 & $.17^{*}$ & -.04 & .01 & .06 & $.19^{* *}$ & $-.15^{*}$ & .09 & $-.23 * *$ & $-.32 * *$ & $.25^{* *}$ & $.16^{*}$ & $.32^{* *}$ & $.16^{*}$ & $.35^{* *}$ & $.54 * *$ & -- & \\
\hline 21 & F contr cumplimiento & 3.23 & .97 & $.18^{* *}$ & -.06 & .07 & .05 & $.55^{* *}$ & $.21 * *$ & $.27 * *$ & $.27 * *$ & $.37 * *$ & $-.41 * *$ & $-.14 *$ & $-.54 * *$ & $-.29 * *$ & $.74 * *$ & $.67^{* *}$ & $.31 * *$ & $.56^{* *}$ & $.47^{* *}$ & $.23 * *$ & $.25 * *$ & -- \\
\hline 22 & F contr descumplimiento & 2.50 & 1.06 & -.09 & -.01 & -.07 & .09 & $-.31 * *$ & $-.17 *$ & $-.14 *$ & -.10 & $-.18 * *$ & $.21^{* * *}$ & $.21 * *$ & $.36^{* *}$ & $.15^{*}$ & $-.43^{* *}$ & $-.46 * *$ & -.03 & $-.27 * *$ & $-.22^{* *}$ & .02 & .00 & $-.59 * *$ \\
\hline
\end{tabular}

Nota. $\mathrm{N}=214$, I: Incremento, F: Fuerza, contr: contractual, const: constituyente, comp: comportamental. * $p<.05$ (bilateral), $* * p<.01$ (bilateral) 


\section{Confirmación de hipótesis}

En la Tabla 3 se muestran los modelos de regresión empleados para verificar las hipótesis de predicción. En el primer paso (Modelo 1) fueron incluidas las variables sociodemográficas (edad, nivel educativo, jerarquía y tiempo de permanencia) para controlar su efecto sobre las variables dependientes. Edad mostró estar significativamente asociada a engagement y al incremento de recursos sociales. Nivel de jerarquía alcanzó resultados significativos únicamente en relación al incremento de demandas desafiantes. Tanto el nivel educativo, como el tiempo de permanencia, no lograron resultados significativos con ninguna de las variables dependientes. En el segundo paso (Modelo 2) se adicionaron las variables independientes (aspectos individuales, contextuales y organizacionales), siguiendo el método paso a paso.

Los análisis de regresión muestran que los aspectos individuales no predicen engagement ni job crafting cuando se controla el efecto de las variables sociodemográficas. Por esto, se rechazan las hipótesis 1 y 2.

Asimismo, las regresiones evidenciaron que la fuerza alternativa predice positiva y significativamente engagement $(\beta=.17, p<.001)$ por lo que se acepta parcialmente la hipótesis 3. De la misma forma, la hipótesis 4 es parcialmente aceptada ya que la dimensión amigos de la fuerza normativa predice el incremento de recursos estructurales y sociales $(\beta=-.15, p<.05$ y $\beta$ $=.20, p<.01$, respectivamente) y la fuerza alternativa predice positivamente el incremento de recursos estructurales y demandas desafiantes $(\beta=.31, p<.001 \mathrm{y} \beta=.19, p<.01$, respectivamente).

Finalmente, respecto a las hipótesis 5 y 6 . Los resultados muestran significativamente que las fuerzas afectivas $(\beta=.41, p<.001)$, calculativa $(\beta=.30, p<.001)$, comportamental psicológica $(\beta=.13, p<.05)$ y tangible $(\beta=.11, p<.05)$ predicen de forma positiva engagement. Por lo que se acepta parcialmente la hipótesis 5. Sobre la hipótesis 6 , las fuerzas calculativa $(\beta=.25, p<.001, \beta=.28, p<.001$ y $\beta=.18, p<.01$ para incremento de recursos estructurales, incremento de recursos sociales e incremento demandas desafiantes respectivamente), constituyente-compañeros $(\beta=.26, p<.001$ para incremento de demandas desafiantes), constituyente-supervisores $(\beta=.36, p<.001$ para incremento de recursos sociales) y comportamental-psicológico $(\beta=.15, p<.05$ y $\beta=.14, p<.05$ para incremento de recursos estructurales e incremento de demandas desafiantes respectivamente) mostraron resultados de predicción significativos para job crafting, por lo que se acepta parcialmente. 
Predictores de engagement en el trabajo y job crafting

Tabla 3.

Resultados de los análisis de regresión

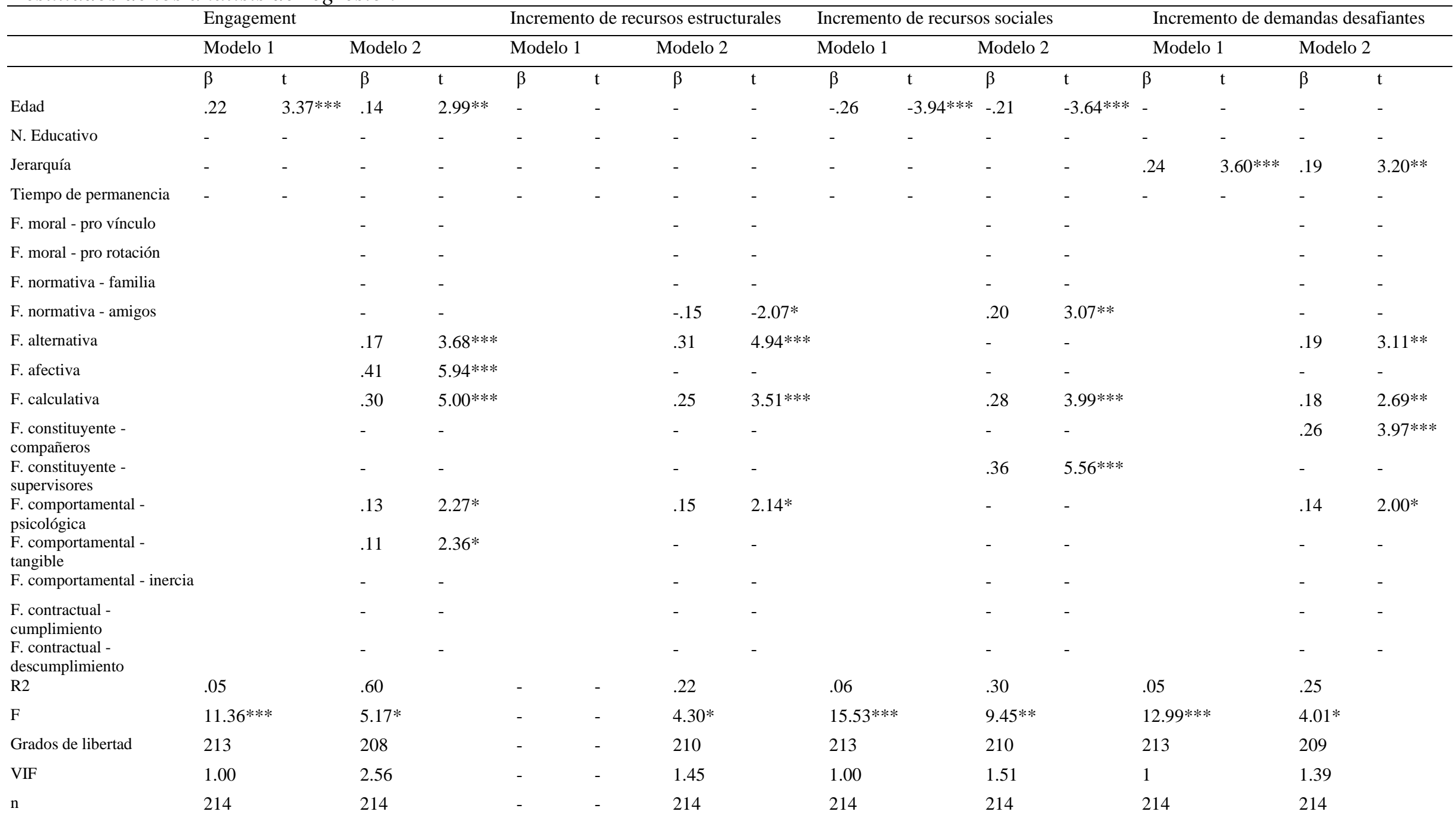

Notas: $* p<.05, * * p<.01, * * * p<.001, \mathrm{~N}:$ Nivel, F: Fuerza, -: Resultado no significativo. 


\section{Discusión}

El presente estudio tuvo como objetivo principal explorar qué aspectos individuales, contextuales y organizacionales influencian engagement y job crafting. Basados en las definiciones de la teoría de demandas y recursos laborales (Demerouti et al., 2001) y en combinación con el modelo de las fuerzas motivacionales para permanencia y turnover de Maertz \& Griffeth (2004). Nuestros resultados fortalecen la idea de que existen situaciones contextuales, además de las organizacionales tradicionalmente asociadas, que tienen impacto en resultados positivos dentro de las empresas. Debajo discutimos los resultados del estudio con mayor detalle.

\section{Efecto de variables sociodemográficas sobre engagement y job crafting}

Para los fines del estudio se controló el efecto de la edad, el nivel educativo, la jerarquía y el tiempo de permanencia ya que diversos estudios (Berdicchia, Nicolli \& Masino, 2016; Petrou et al., 2015; Vasquez et al., 2015; Yim, Choi \& Park, 2015) y teorías en el campo de la psicología organizacional refieren relaciones con nuestras variables.

La edad tuvo un efecto positivo sobre engagement en el trabajo, así como lo reportado por Vasquez et al. (2015), quienes encontraron que, a mayor edad, mayores niveles de engagement. Asimismo, se evidenció el efecto negativo de este sobre el incremento de recursos sociales de job crafting. Es decir, conforme las personas van ganando edad son menos los esfuerzos dirigidos hacia la búsqueda de soporte social y retroalimentación. Esta reducción refuerza lo sugerido por Kanfer y Ackerman (2004), quienes notaron que los trabajadores más jóvenes, en comparación a los mayores, son orientados al aprendizaje, lo que podría explicar su mayor inclinación a este tipo de job crafting. Kooij, Tims, y Kanfer (2015) mencionan también que los trabajadores mayores prefieren interacciones sociales que afirmen sus competencias a interacciones que les ofrezcan oportunidades futuras de desarrollo de carrera.

La jerarquía tuvo un efecto positivo sobre el incremento de demandas desafiantes de job crafting. Conforme se posea una posición de mayor jerarquía, se invertirá más en acciones orientadas a este tipo de job crafting. Eso está en consonancia con hallazgos sobre la relación positiva entre el job crafting orientado a la extensión de tareas con los grados de autonomía (Petrou, Demerouti \& Xanthopoulou, 2016).

Es importante notar que el nivel educativo ni el tiempo de permanencia alcanzaron resultados significativos con ninguna de las variables en estudio. Lo que sugiere que todas las personas, independientemente de los recursos académicos o el tiempo que tengan dentro de una organización, pueden modificar activamente sus ambientes laborales para que estos se conviertan en espacios más satisfactorios y con mayor significancia personal.

\section{Predictores contextuales}

La fuerza alternativa, dentro de los aspectos contextuales predijo de forma positiva, tanto engagement como job crafting. Esta medida, si bien es un reflejo de la autoeficacia con respecto a la capacidad propia de conseguir un empleo nuevo, está supeditada a la percepción que tenemos del mercado laboral en el que estamos inmersos (Oliveira et al., 2016). Los resultados refuerzan la postura de que un profesional empleable no necesariamente busca nuevas alternativas sino por el contrario, esto contribuye al propio sentido de éxito y satisfacción laboral (Guest \& Rodrigues, 2015). Por otro lado, de acuerdo con la teoría de conservación de recursos, al invertir los recursos propios (formas de job crafting) en el aumento de la empleabilidad, la persona reduce el riesgo de quedar desempleada, al tiempo que aumenta la posibilidad de conseguir un mejor trabajo con mejores oportunidades de aprendizaje y crecimiento, lo que 
finalmente mejora su estado de engagement y conduce a una espiral de ganancia (Salanova et al., 2010). Del mismo modo, con respecto a engagement, los resultados se relacionan con lo hallado por Schaufeli, Dijkstra y Vázquez (2013), quienes reportaron relaciones positivas entre la autoeficacia y el bienestar afectivo en el trabajo.

La fuerza normativa, específicamente la dimensión que refiere a los amigos fuera de la organización, alcanzó potencial significativo para predecir el incremento de recursos estructurales y sociales. Así, conforme el trabajador perciba que su entorno amical, externo a la organización, no está de acuerdo con que este continúe en su actual trabajo, este invertirá menos esfuerzos en el incremento de sus recursos estructurales (i.e., aprender cosas nuevas, involucrarse en nuevos proyectos) y por el contrario buscará aumentar sus recursos sociales (i.e., búsqueda de soporte social y retroalimentación) para compensar el soporte faltante por parte de los amigos externos, con la creación de nuevas redes internas.

\section{Predictores organizacionales}

Cuatro de los cinco aspectos organizacionales evaluados obtuvieron resultados significativos como predictores de engagement y job crafting. Eso respalda lo encontrado por Macey y Schneider (2008), referente a que la experiencia positiva implicada en sentir engagement tiene como trasfondo la configuración del trabajo. Respecto a job crafting, Wrzesniewski y Dutton (2001), señalan también que no es suficiente facilitar oportunidades para promover estos comportamientos. Esto en conjunto, fortalece además lo propuesto en el modelo de las características del puesto de Hackman y Oldman (1975), las organizaciones pueden actuar sobre diversos aspectos del trabajo para incentivar la resignificación de este, la responsabilidad propia sobre los objetivos de la organización y otros fenómenos positivos.

La fuerza afectiva, que refleja sentimientos positivos de comodidad o placer hacia la organización, tiene como desencadenante lógico el aumento de engagement. Hacia la cual efectivamente mostró poder predictivo. Como mencionan Schaufeli y Bakker (2010), engagement tiene una connotación que refiere al involucramiento, compromiso, pasión, foco y energía, los cuales implícitamente hacen referencia a afectos positivos y al bienestar. Incluso Salanova et al., (2010) mencionan los trabajadores en el estado de engagement consideran que están en situaciones placenteras, aun teniendo que lidiar con los desafíos y demandas laborales.

La fuerza calculativa, es decir, la percepción de oportunidades de alcanzar los objetivos profesionales de vida a través de la organización, se mostró como un aspecto predictor tanto de engagement como de todas las dimensiones de job crafting. Los resultados muestran que, si la evaluación sobre desarrollo o crecimiento son positivas, los individuos van a sentir más engagement y van a invertir fuerzas y energías para involucrarse en aprender cosas nuevas, en buscar retroalimentación y en proyectos emergentes (incremento de recursos estructurales, sociales y demandas desafiantes). Al respecto, Baruch (2006) menciona que las organizaciones dejan de significar control respecto al desarrollo profesional de las personas, para convertirse en soporte y desarrollo, como un medio que posibilite el éxito de sus trabajadores.

La fuerza constituyente, es decir la calidad de las relaciones mantenidas en el contexto laboral, fue predictor de job crafting en sus formas de incremento de recursos sociales e incremento de demandas desafiantes. Las relaciones positivas con los compañeros estimulan el incremento de demandas desafiantes, mientras que las relativas al jefe o supervisor impactan sobre el incremento de recursos sociales. Estos resultados muestran que las relaciones interpersonales afectan la forma en cómo los individuos alteran sus condiciones de trabajo. Las personas en general invierten sus recursos en conservar o mejorar sus relaciones de apoyo (Allen, Shore \& Griffeth, 2003) lo que explica que, ante medios donde existan relaciones interpersonales positivas, las personas se involucraran en formas de job crafting que impliquen contacto con otros. Por otro lado, el estudio de Parker, Willliams y Turner (2006) muestra que 
sentir confianza en los compañeros conduce a las personas a involucrarse en comportamientos proactivos. Esto nos lleva a inferir que en espacios donde las relaciones interpersonales son negativas, existe menos orientación para involucrarse, por iniciativa propia, en comportamientos de rediseño del trabajo.

Finalmente, la fuerza comportamental evidenció ser significativamente importante para predecir tanto engagement como dos formas de job crafting (incremento de recursos estructurales y demandas desafiantes). Estas fuerzas comportamentales, específicamente la psicológica y la tangible, refieren al deseo de evitar el costo psicológico y explícito que viene asociado al retiro de la organización (Oliveira, 2016). Es posible que frente a la crisis económica y el reducido número de agencias de comunicación en la ciudad donde se realizó el estudio, los participantes hayan percibido que salir de la organización implicaría grandes pérdidas. Esa percepción, llevaría a mayores niveles de engagement y job crafting, sobre todo de actividades capaces de tornar el trabajo más interesante y desafiante.

Queda clara entonces que, los aspectos afectivos, las posibilidades de crecimiento, las relaciones interpersonales y los costos asociados a la pérdida del trabajo, no solo son importantes como promotores de la satisfacción en el trabajo, o la motivación relacionada a esta, como exponen ampliamente los modelos de motivación laboral de Herzberg y McClelland de la literatura clásica organizacional. Sino que todos estos generan además fenómenos con altas cargas energéticas y orientación a la acción como son engagement y job crafting.

\section{Predictores individuales}

El modelo de las ocho fuerzas motivacionales para permanencia y turnover (Maertz \& Griffeth, 2004) contempla la fuerza moral como un reflejo de los valores individuales sobre lo positivo o negativo que significa el cambio constante entre empleadores (Oliveira et al., 2016). Se evaluó la posibilidad de que esta valoración moral pudiese impactar en el estado de engagement o en los comportamientos de job crafting. Sin embargo, en ninguno de los modelos de regresión, representaron variables importantes para la explicación de estos fenómenos.

\section{Implicancias teóricas y prácticas}

El presente estudio contribuye y amplía las investigaciones sobre engagement y job crafting en cuatro formas. Primero, extiende la literatura sobre estas variables y su relación con percepciones o motivadores individuales, en consonancia con los estudios de Esteves y Lopes (2001), y Petrou et al. (2015). Ya que, en esencia la medida de las ocho fuerzas motivacionales son un reflejo de cómo los trabajadores perciben diversos aspectos relacionados al trabajo.

Segundo, los resultados mostrados evidencian cómo aspectos vinculados a la permanencia o salida de los trabajadores impactan también, de manera diferenciada, en el estado afectivo de engagement y en la forma en cómo los trabajadores alteran sus condiciones laborales (job crafting). Estos hallazgos orientan a los profesionales en gestión del recurso humano sobre qué cuestiones deben ser atendidas al momento de implementar políticas que vayan más allá del incentivo de estados pasivos de bienestar como es la satisfacción en el trabajo. Específicamente, prestando atención a los aspectos organizacionales con potencial de predicción. Por ejemplo, los análisis mostraron que tener planes de desarrollo profesional claros (reflejo de la fuerza calculativa) incrementa los niveles de engagement en el trabajo y los comportamientos de job crafting, lo que finalmente repercutirá en mayores niveles de desempeño y contribuirá por consecuencia a la estrategia del negocio.

Así, los planes de sucesión, la identificación y desarrollo de colaboradores con alto potencial y los diálogos individuales sobre la propia carrera, son buenas prácticas que contribuirían con el incremento de estados y comportamientos positivos en el trabajo 
(engagement y job crafting). Del mismo modo, la incorporación de programas de entrenamiento y de desarrollo, incluso de job crafting, incrementan la confianza y autoeficacia de las personas, los motiva a que sientan más engagement (Anitha, 2014) y tengan más herramientas y recursos para invertir a través de comportamientos de job crafting.

Tercero, y en estrecha relación con lo anteriormente expuesto, nuestros resultados brindan informaciones valiosas al área de comunicación y marketing. Esta área tuvo nuestra atención especial ya que, como comentan Carvalho et al. (2016), se percibe que es un sector que viene modificando sus prácticas internas para poder atraer y mantener sus talentos. Todavía hay pocos estudios sobre fenómenos psicológicos en profesionales del área, a pesar de que el sector ha movido más de 15 mil millones de reales en los últimos años (Sacchitiello, 2019). En conjunto, estos factores pueden ser algunos de los responsables de la experiencia frecuente de un entorno de sobrecarga de trabajo y estrés profesional (Sarquis \& Ikeda, 2007). Por lo tanto, los resultados obtenidos deben tomarse como una foto del momento que está viviendo el área, para de esta forma proponer planes de acción con miras al mejoramiento de las condiciones de sus empleados. Estos deben tener como foco de trabajo la promoción de la identificación afectiva en relación con la organización, promoción y aclaración sobre las posibilidades de crecimiento profesional, favoreciendo las relaciones interpersonales positivas para promover un ambiente de trabajo que se perciba como poco deseable para abandonar.

Cuarto, contribuye al entendimiento contextualizado de estas variables en un momento específico del país (Brasil). Considerando que las relaciones sociales y económicas envuelven cualquier tipo de comportamiento organizacional, al centrarnos en un grupo y mercado específico (área de comunicación y marketing) y sus particularidades, podemos tener una visión más precisa sobre los hechos que motivan a los profesionales de este grupo ocupacional.

\section{Limitaciones y futuros pasos}

Así como otras investigaciones, el presente estudio contempla ciertas limitaciones. Primero, por ser un estudio de tipo transversal su potencial de generalización es limitado con respecto a estudios longitudinales. Futuras investigaciones podrían adoptar medidas en diferentes tiempos para comprender el efecto real de estos aspectos (individuales, contextuales y organizacionales) sobre engagement y job crafting.

Las medidas empleadas para los análisis surgieron a partir de cuestionarios de auto reporte, los cuales son afectados por la subjetividad de cada individuo. Por otro lado, ya que los cuestionarios fueron analizados dentro de un contexto organizacional sus resultados podrían obedecer a un intento por mostrar los aspectos positivos de esta.

Dentro de los aspectos individuales solo fueron considerados los valores y creencias sobre permanecer o retirarse de la organización. Futuras investigaciones podrían indagar más a detalle en la relación de engagement y job crafting con otros aspectos individuales como autoeficacia, personalidad proactiva, resiliencia en el trabajo, entre otros.

La evaluación de job crafting incluye únicamente tres tipos (incremento de recursos estructurales, recursos sociales y demandas desafiantes) la dimensión de reducción de demandas que figura en diversas evaluaciones del constructo no alcanzó resultados significativos cuando se realizó la adaptación y validación del instrumento en el contexto brasileño (Chinelato et al., 2105). Es decir, la población no identificó los comportamientos de job crafting de tipo expansivos como pertenecientes al mismo grupo que aquellos enfocados en reducir demandas en sus trabajos. Este fenómeno merece futuras investigaciones orientadas a esclarecer si esto se debe a algunas dinámicas diferenciadoras del contexto a al instrumento en sí. Finalmente, nuestro estudio se enfocó en un área ocupacional única -comunicación y marketing- lo que podría también limitar la generalización de estos resultados. Por lo que se sugiere replicar la presente investigación para ampliar la comprensión de los fenómenos. 


\section{Referencias}

Airila, A., Hakanen, J. J., Schaufeli, W. B., Luukkonen, R., Punakallio, A., \& Lusa, S. (2014). Are job and personal resources associated with work ability 10 years later? The mediating role of work engagement. Work \& Stress, 28, 87-105. doi:10.1080/02678373.2013.872208

Anitha J., (2014). Determinants of employee engagement and their impact on employee performance. International Journal of Productivity and Performance Management, 63(3), 308-323. doi: 10.1108/IJPPM-01-2013-0008

Allen, D. G., Shore, L. M., \& Griffeth, R. W. (2003). The role of perceived organizational support and supportive human resource practices in the turnover process. Journal of Management, 29, 99-118. doi:10.1177/014920630302900107

Bakker, A.B. \& Bal, P.M. (2010). Weekly work engagement and performance: a study among starting teachers. Journal of Occupational and Organizational Psychology, 83, 189-206. doi:10.1348/096317909X402596

Bakker, A. B. \& Demerouti, E. (2014). Job demands - resources theory. En: C. Cooper \& P. Chen (Eds.), Wellbeing: A complete reference guide (pp. 37-64). Chichester, UK: WileyBlackwell.

Bakker, A. B., \& Demerouti, E. (2016). Job Demands-Resources Theory: Taking Stock and Looking Forward. Journal of Occupational Health Psychology. Avance de publicación online. doi:10.1037/ocp0000056

Bakker, A. B., Demerouti, E., \& Sanz-Vergel, A. I. (2014). Burnout and work engagement: The JD-R approach. Annual Review of Organizational Psychology and Organizational Behavior, 1(1), 389-411. doi: 10.1146/annurev-orgpsych-031413-091235

Bakker, A. B., Rodríguez-Muñoz, A., \& Sanz-Vergel, A. I. (2016). Modelling job crafting behaviours: Implications for work engagement. Human Relations, 69(1), 169-189. doi: $10.1177 / 0018726715581690$

Bakker, A. B., \& Schaufeli, W. B. (2008). Positive organizational behavior: engaged employees in flourishing organizations. Journal of Organizational Behavior, 29, 47-g

Bakker, A. B., Schaufeli, W. B., Leiter, M. P., \& Taris, T. W. (2008). Work engagement: An emerging concept in occupational health psychology. Work \& Stress, 22, 187-200. doi: $10.1080 / 02678370802393649$

Bakker, A. B., \& Xanthopoulou, D. (2013). Creativity and charisma among female leaders: The role of resources and work engagement. International Journal of Human Resource Management, 24, 2760-2779. doi: 10.1080/09585192.2012.751438

Baruch, Y. (2006). Career development in organizations and beyond: Balancing traditional and contemporary viewpoints. Human resource management review, 16(2), 125-138. doi: 10.1016/j.hrmr.2006.03.002

Berdicchia, D., Nicolli, F., \& Masino, G. (2016). Job enlargement, job crafting and the moderating role of self-competence. Journal of Managerial Psychology, 31(2), 318-330. doi:10.1108/JMP-01-2014-0019

Carvalho, C. M., Alves, D. A., \& Machado, A. R. (2016). As novas gerações e o trabalho publicitário. En: E. Freitas, J.A. Saraiva, \& G. Haubrich (Eds.), Diálogos Interdisciplinares: Cultura, Comunicação e Diversidade no Contexto Contemporâneo. (pp. 201-213). Nuevo Hamburgo, RS: Feevale.

Carvalho, C. M., \& Christofoli, M. P. (2015). O campo publicitário, a agência e a noção de aceleração do tempo: questões iniciais para pensar novos modelos e negócios na prática do mercado publicitário. Sessões do Imaginário, 20(34), 91-99. doi:10.15448/19803710.2015 .2 
Chinelato, R. S. C., Ferreira, M. C., \& Valentini, F. (2015). Evidence of validity of the Job Crafting Behaviors Scale. Paidéia (Ribeirão Preto), 25(62), 325-332. doi:10.1590/198243272562201506

Culbertson, S. S., Mills, M. J., \& Fullagar, C. J. (2012). Work engagement and work-family facilitation: Making homes happier through positive affective spillover. Human Relations, 65(9), 1155-1177. doi:10.1177/0018726712440295

Demerouti, E. (2014). Design your own job through job crafting. European Psychologist, 19, 237-247. doi:10.1027/1016-9040/a000188

Demerouti, E., Bakker, A. B., \& Gevers, J. M. (2015). Job crafting and extra-role behavior: The role of work engagement and flourishing. Journal of Vocational Behavior, 91, 87-96. doi:10.1016/j.jvb.2015.09.001

Demerouti, E., Bakker, A. B., Nachreiner, F., \& Schaufeli, W. B. (2001). The job demandsresources model of burnout. Journal of Applied psychology, 86(3), 499-512. doi:10.1037/0021-9010.86.3.499

Esteves, T., \& Lopes, M. P. (2001). Crafting a Calling: The Mediating Role of Calling Between Challenging Job Demands and Turnover Intention. Journal of Career Development, 44(1), 34-48. doi:10.1177/0894845316633789

Guest, D. E. \& Rodrigues, R. (2015). Career control. En: A. De Vos \& B. Van der Heijden (Eds), Handbook of Research on Sustainable Careers (pp. 205-222). Cheltenham: Edward Elgar Publishing.

Hakanen, J. J., Peeters, M. C., \& Schaufeli, W. B. (2017). Different types of employee wellbeing across time and their relationships with job crafting. Journal of occupational health psychology. Avance de publicación online. doi:10.1037/ocp0000081

Hakanen, J.J., Perhoniemi, L., Toppinen-Tammer, S. (2008). Positive gain spirals at work: from job resources to work engagement, personal initiative and work-unit innovativeness. Journal of Vocational Behavior, 73(1), 78-91. doi:10.1016/j.jvb.2008.01.003

Hakanen, J. J., \& Schaufeli, W. B. (2012). Do burnout and work engagement predict depressive symptoms and life satisfaction? A three-wave seven-year prospective study. Journal of Affective Disorders, 141, 415-424. doi:10.1016/j.jad.2012.02.043

Holtom, B. C., Mitchell, T. R., Lee, T. W., \& Eberly, M. B. (2008). Turnover and retention research: A glance at the past, a closer review of the present, and a venture into the future. Academy of Management Annals, 2, 231-274. doi:10.1080/19416520802211552

Janssen, O., Van de Vliert, E., \& West, M. (2004). The bright and dark sides of individual and group innovation: A Special Issue introduction. Journal of Organizational Behavior, 25, 129-145. doi:10.1002/job.242

Kanfer, R., \& Ackerman, P. L. (2004). Aging, adult development, and work motivation. The Academy of Management Review, 29, 440-458.

Kooij, D. T., Tims, M., \& Kanfer, R. (2015). Successful aging at work: The role of job crafting En: P. Bal, D. Kooij, \& D. Rousseau (Eds.) Aging workers and the employee-employer relationship (pp. 145-161). Londres: Springer. doi:10.1007/978-3-319-08007-9_9

Macey, W. H., \& Schneider, B. (2008). The meaning of employee engagement. Industrial and Organizational Psychology, 1, 3-30. doi:10.1111/j.1754-9434.2007.0002.x

Maertz, C.P. Jr., \& Griffeth, R.W. (2004). Eight motivational forces and voluntary turnover: A theoretical synthesis with implications for research. Journal of Management, 30(5), 667683. doi:10.1016/j.jm.2004.04.001

Lascbinger, H.K.S., Wong, C.A., Greco, P., 2006. The impact of staff nurse empowerment on person-job fit and work engagement/burnout. Nursing Administration Quarterly, 30(4), 358-367.

Oldham, G. R., \& Cummings, A. (1996). Employee creativity: Personal and contextual factors at work. Academy of Management Journal, 39, 607-634. doi:10.2307/256657 
Oliveira, M. Z. D., Beria, F. M., \& Gomes, W. B. (2016). Validity Evidence for the Turnover and Attachment Motives Survey (TAMS) in a Brazilian Sample. Paidéia (Ribeirão Preto), 26(65), 333-342. doi:10.1590/1982-43272665201604

Parker, S. K., Williams, H. M., \& Turner, N. (2006). Modeling the antecedents of proactive behavior. Journal of Applied Psychology, 91, 636-652. doi: 10.1037/0021-9010.91.3.636

Petrou, P., Demerouti, E. \& Schaufeli, W. B. (2015). Job Crafting in Changing Organizations: Antecedents and Implications for Exhaustion and Performance. Journal of Occupational Health Psychology, 20(4), 470-480. doi:10.1037/a0039003

Petrou, P., Demerouti, E., \& Xanthopoulou, D. (2016). Regular Versus Cutback-Related Change: The Role of Employee Job Crafting in Organizational Change Contexts of Different Nature. International Journal of Stress Management. Avance de publicación online. doi:10.1037/str0000033

Rich, B.L., Lepine, J.A., Crawford, E.R., 2010. Job engagement: antecedents and effects on job performance. Academy of Management Journal, 53(3), 617-635. doi:10.5465/AMJ.2010.51468988

Rios, M. C., Lula, A. M., do Amaral, N. D. A., \& Bastos, A. V. B. (2014). Contratos psicológicos e comprometimento: o mapeamento cognitivo dos construtos junto a profissionais de RH. Acta Científica. Ciências Humanas, 1(16), 9-24.

Sacchitiello, B. (2019, abril 10). Cenp-Meios: compra de mídia em 2018 foi praticamente igual à de 2017. Meio \& Mensagem. Recuperado de https://www.meioemensagem.com.br/home/midia/2019/04/10/mercado-publicitariomovimenta-r-165-bilhoes-em-2018.html

Salanova, M., Schaufeli, W. B., Xanthopoulou, D., \& Bakker, A. B. (2010). The gain spiral of resources and work engagement: Sustaining a positive worklife. En: A. B. Bakker \& M. P. Leiter (Eds.), Work engagement: A handbook of essential theory and research (pp. 118-131). New York: Psychology Press

Sarquis, A. B., \& Ikeda, A. A. (2007). A prática de posicionamento de marca em agências de comunicação. Revista de Negócios, 12(4), 55-70.

Schaufeli, W. B., \& Bakker, A. B. (2010). Defining and measuring work engagement: Bringing clarity to the concept. En: A. B. Bakker \& M. P. Leiter (Eds.), Work engagement: A handbook of essential theory and research (pp. 10-24). Nueva York: Psychology Press.

Schaufeli, W. B., Bakker, A. B., \& Salanova, M. (2006). The measurement of work engagement with a short questionnaire: A cross-national study. Educational and Psychological Measurement, 66, 701-716. doi:10.1177/0013164405282471

Schaufeli, W. B., Dijkstra, P., \& Vazquez, A. C. (2013). Engajamento no trabalho. São Paulo: Casa do Psicólogo.

Shin, S. J., \& Zhou, J. (2003). Transformational leadership, conservation, and creativity: Evidence from Korea. Academy of Management Journal, 46, 703-714. doi: $10.2307 / 30040662$

Scott, S. G., \& Bruce, R. A. (1994). Determinants of innovative behavior: A path model of individual innovation in the workplace. Academy of Management Journal, 37, 580-607. doi: $10.2307 / 256701$

Slemp, G. R., \& Vella-Brodrick, D. A. (2014). Optimizing employee mental health: the relationship between intrinsic need satisfaction, job crafting, and employee well-being. Journal of Happiness Studies, 15(4), 957-977. doi:10.1007/s 10902-013-9458-3

Tims, M., Bakker, A. B., \& Derks, D. (2013). The impact of job crafting on job demands, job resources, and well-being. Journal of occupational health psychology, 18(2), 230-240. doi: $10.1037 / \mathrm{a} 0032141$ 
Vazquez, A. C. S., Magnan, E. D. S., Pacico, J. C., Hutz, C. S., \& Schaufeli, W. B. (2015). Adaptation and Validation of the Brazilian Version of the Utrecht Work Engagement Scale. Psico-USF, 20(2), 207-217. doi: 10.1590/1413-82712015200202

Wingerden, J. V., Bakker, A. B., \& Derks, D. (2016). A test of a job demands-resources intervention. Journal of Managerial Psychology, 31(3), 686-701. doi:10.1108/JMP-032014-0086

Wrzesniewski, A., \& Dutton, J. E. (2001). Crafting a job: Revisioning employees as active crafters of their work. Academy of management review, 26(2), 179-201. doi:10.5465/AMR.2001.4378011

Yim, S., Choi, A., \& Park, K. (2015). Effects of Employee Value Proposition and Proactive Personality on Job Crafting: South Korean Professional Assistants' Case. International Information Institute (Tokyo). Information, 18(11), 4579-4585.

Contribución de los autores: a) Concepción y diseño del trabajo; b) Adquisición de datos; c) Análisis e interpretación de datos; d) Redacción del manuscrito; e) revisión crítica del manuscrito.

J.A. ha contribuido en a,b,c,d,e ; P.O. b,c,d,e en ; B.S.T en b,c,d,e;M.Z.O. en a,b,c,d,e.

Editora científica responsable: Dra. Cecilia Cracco 